\title{
Collapse Modeling of a Masonry Arch Dam Using the Cohesive Interface Elements
}

\author{
Jianwen Pan \\ State Key Laboratory of Hydroscience and Engineering, Tsinghua University, Beijing 100084, China \\ Correspondence should be addressed to Jianwen Pan; panjianwen@tsinghua.edu.cn
}

Received 11 February 2015; Revised 10 May 2015; Accepted 11 May 2015

Academic Editor: Evangelos J. Sapountzakis

Copyright (C) 2015 Jianwen Pan. This is an open access article distributed under the Creative Commons Attribution License, which permits unrestricted use, distribution, and reproduction in any medium, provided the original work is properly cited.

\begin{abstract}
A finite element (FE) approach with zero-thickness cohesive interface elements is presented to simulate collapse of continuum structures. The element removal technique merged with the general contact algorithm is adapted in the FE approach to achieve modeling for a transition from continua to discontinua, that is, fracture, fragmentation, and collapse. Collapse process of Meihua masonry arch dam, which is a famous disaster in dam engineering in China, is simulated and the failure mechanism is studied. The collapse process obtained from the presented procedure coincides with the field observation after the dam failure. The failure of Meihua arch dam can be attributed to reducing shear strength of the peripheral joint between the dam body and the concrete pedestal by daubing a layer of asphalt there. With low sliding resistance strength, the masonry dam body may slide upwards along the peripheral joint under hydrostatic pressure, leading to weakening of the arch action, fracturing, and final collapse of the dam.
\end{abstract}

\section{Introduction}

Dam collapse results in catastrophe subsequences of a sudden release of reservoir and the following flood may cause losses of life and property downstream. Predictive modeling of the dam collapse process and scenario, based on which the catastrophe floods can be simulated, is necessary for taking perhaps protective or procedural measures to reduce the risk to human life and property.

Many efforts have been devoted to simulation of fracturing in concrete dams. The damage based model is a common method for recent cracking analysis of concrete dams in the framework of finite element (FE) method. This type of methods can provide appropriate results of cracking initiation and subsequent propagation in large dams [1-7]. Besides, the scaled boundary finite element method (SBFEM) [8] and the extended finite element method (XFEM) coupled with the damage mechanics approach [9] have been, respectively, proposed to simulate crack propagation in concrete gravity dams subjected to gravity and overflow pressure. Several fracture modeling approaches are compared with simulation of cracking behavior of concrete gravity dams under overloading conditions [10]. The continuum mechanical approaches mentioned above, within the context of small-strain theory, can only provide fracture patterns of concrete dams but do not have the capability of modeling collapse process of the structure or predicting collapse scenarios. The discrete element model (DEM) has an inherent advantage in collapse modeling of structures under hazardous loads. DEM has been applied to simulation of collapse process of the fractured Koyna Dam under earthquake [11]. Although DEM is capable of simulation of collapse behavior of structures, the obtained stress distribution in the structure before its failure is insufficiently accurate due to the contact force potentials between adjacent discrete blocks. A numerical modeling is therefore required for simulation of progressive collapse of structures subjected to extreme loads.

Cohesive crack models $[12,13]$ avoid stress singularity ahead the crack tip in linear elastic fracture mechanics and have been widely applied to fracture modeling of brittle materials in the context of the FEM [14-16]. Saouma [17] presented a comprehensive perspective on applications of fracture mechanics to cementitious materials. Cohesive interface element (CIE) approach, whose most distinct advantage is the simplicity of implementation into existing FE codes, is one of the first models capable of modeling discrete crack propagation $[18,19]$. The approach by Xu and Needleman [20], in which the CIEs are introduced at the beginning of 


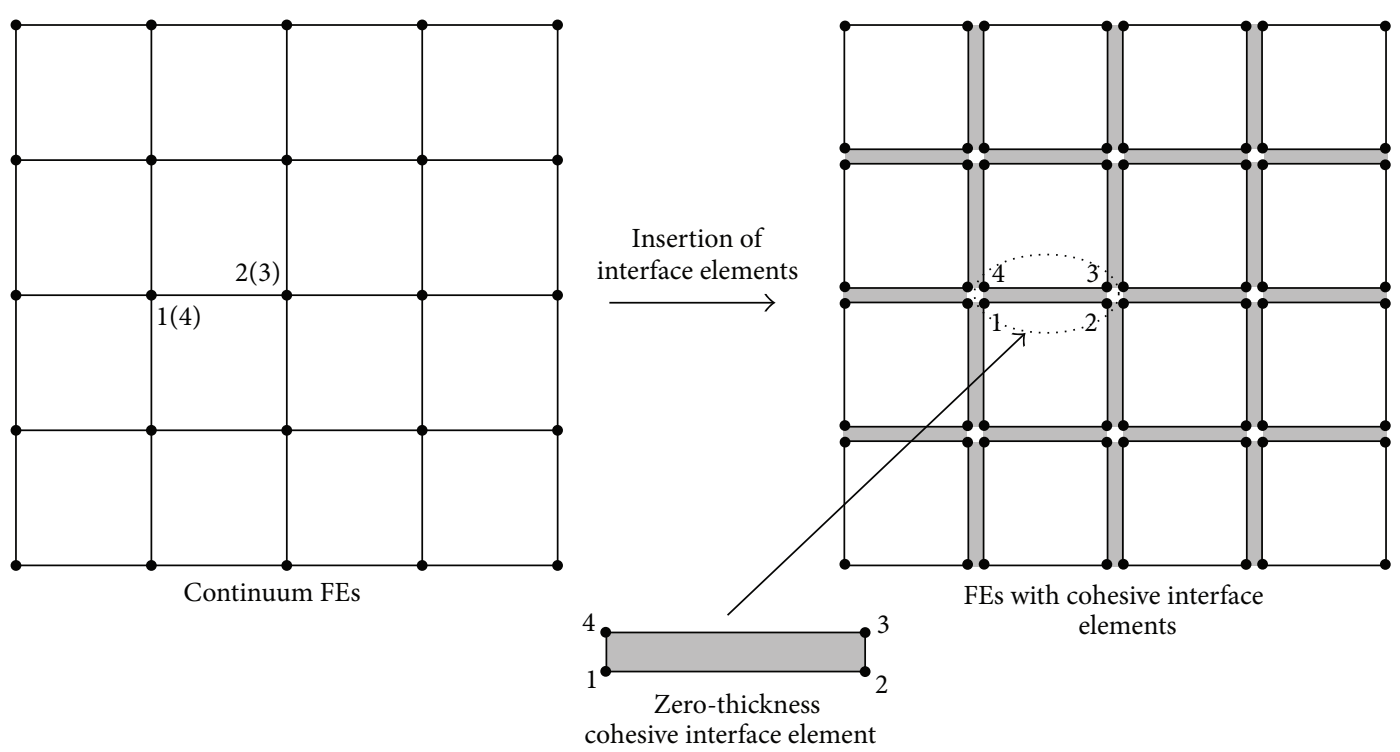

Figure 1: Discretization of solid into FEs with zero-thickness CIEs.

the simulation, is applied to modeling dynamic fracture and problems involving numerous cracks with complex fracture patterns due to its simplicity and efficiency $[21,22]$.

In this study, the element removal technique merged with the general contact algorithm is introduced in the CIE approach. Cracks developing in the solid medium are represented by the failure of CIEs. The CIEs are deleted as they completely fail, and thus the cracks divide the solid medium into pieces of deformable bodies which can be further fragmented. The general contact algorithm is then activated to govern the interaction between the deformable bodies. The modeling for a transition from continua to discontinua, that is, fracture, fragmentation, and collapse, is achieved using the FE approach with CIEs. The approach is applied to collapse modeling of Meihua masonry arch dam which is a famous dam disaster in China. The failure mechanism of the arch dam is discussed.

\section{Numerical Modeling}

2.1. Cohesive Interface Elements (CIEs). Consider a twodimensional solid medium. We first discretize it by continuum finite elements (FEs), and then we insert zero-thickness cohesive interface elements (CIEs) along the interfaces of the continuum FEs, as shown in Figure 1.

The continuum FEs behave as a linear elastic material in the simulation. Cracking of the solid is assumed to be occurring and propagating along the interfaces of the continuum FEs, and the response of the CIEs for modeling progressive damage and failure is defined in terms of traction-separation. This study adopts a mixed mode cohesive model, which was proposed by Camanho and Davila [23]. It involves the simultaneous activation of normal and shear displacements discontinuity with respect to the crack and corresponding tractions. Once the damage initiation criterion is met, damage occurs in the CIEs according to the damage evolution law.
The maximum nominal stress criterion is considered, and it is represented as

$$
\max \left(\frac{\left\langle t_{n}\right\rangle}{t_{n 0}}, \frac{t_{s}}{t_{s 0}}, \frac{t_{p}}{t_{p 0}}\right)=1,
$$

where $t_{n}, t_{s}$, and $t_{p}$ are the normal and tangential tractions, respectively; $t_{n 0}, t_{s 0}$, and $t_{p 0}$ are the peak values of the traction when the deformation is either purely normal to the interface or purely in the first or the second shear direction, respectively; and $\langle\cdot\rangle$ denotes the Macaulay bracket $\langle x\rangle=$ $\left\{\begin{array}{l}0, \text { if } x<0 \\ x, \text { if } x>0\end{array}\right.$, being used to signify that a pure compressive deformation does not initiate damage.

The effective displacement of the crack in the CIEs is defined by

$$
\delta_{e}=\sqrt{\left\langle\delta_{n}\right\rangle^{2}+\delta_{s}^{2}+\delta_{p}^{2}}
$$

where $\delta_{n}$ is the normal displacement and $\delta_{s}$ and $\delta_{p}$ are the two local shear displacements of the crack surface. Likewise, the effective traction is written as

$$
t_{e}=\sqrt{\left\langle t_{n}\right\rangle^{2}+t_{s}^{2}+t_{p}^{2}} .
$$

A linear softening traction-separation relation in terms of effective traction and effective displacement shown in Figure 2 is used in this work. The prepeak region represents the elastic part of the cohesive law, whereas the softening portion after the peak load considers the damage occurring in the fracture process zone. Inelastic strain is not considered before the cohesive element is completely damaged.

A scalar damage variable, $d$, is introduced to describe the rate at which the stiffness of CIEs is degraded once the damage initiation criterion is reached. It initially has a value of 0 and monotonically evolves from 0 to 1 upon further loading after the initiation of damage. Considering 


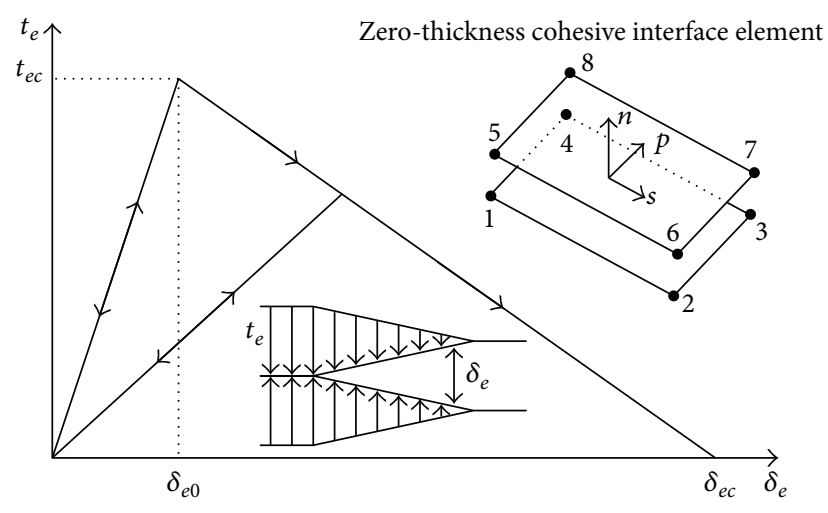

Figure 2: Cohesive law in terms of effective displacement and effective traction.

the evolution of damage under the combination of normal and shear deformation across the interface, the damage variable is defined as

$$
d=\frac{\delta_{e c}\left(\delta_{e \max }-\delta_{e 0}\right)}{\delta_{e \max }\left(\delta_{e c}-\delta_{e 0}\right)}
$$

where $\delta_{e 0}$ is the effective displacement at damage initiation; $\delta_{e c}$ is the effective displacement at complete failure; and $\delta_{e \max }$ denotes the maximum value of the effective displacement attained during the loading history; that is, $\delta_{e \max }=\max \left(\delta_{e}\right)$.

The tractions across the interface are affected by the damage according to

$$
\begin{aligned}
t_{n} & = \begin{cases}(1-d) \bar{t}_{n}, & \bar{t}_{n} \geq 0 \\
\bar{t}_{n}, & \bar{t}_{n}<0,\end{cases} \\
t_{s} & =(1-d) \bar{t}_{s}, \\
t_{p} & =(1-d) \bar{t}_{p},
\end{aligned}
$$

where $\bar{t}_{n}, \bar{t}_{s}$, and $\bar{t}_{p}$ are the stress components predicted by the elastic behavior for the current displacement without damage; that is,

$$
\left[\begin{array}{l}
\bar{t}_{n} \\
\bar{t}_{s} \\
\bar{t}_{p}
\end{array}\right]=\left[\begin{array}{lll}
\bar{k}_{n} & & \\
& \bar{k}_{s} & \\
& & \bar{k}_{p}
\end{array}\right]\left[\begin{array}{l}
\delta_{n} \\
\delta_{s} \\
\delta_{p}
\end{array}\right],
$$

where $\bar{k}_{n}, \bar{k}_{s}$, and $\bar{k}_{p}$ are the initial normal and shear stiffness across the interface, respectively. A large value for the initial stiffness is assumed and the CIEs are inactive before damage initiation criterion is reached.

2.2. Transition from Continua to Discontinua. Structural collapse may occur due to hazardous loading conditions such as earthquakes, impacts, blasts, and floods. In the collapse process, the phenomena can be similar. The continuous structure displays fracture and fragmentation and finally transforms into a pile of rubble.
The element removal technique merged with the general contact algorithm is used to achieve modeling for a transition from continua to discontinua, that is, fracture, fragmentation, and collapse. This procedure is implemented in the explicit FE code ABAQUS [24]. Figure 3 shows the process of the transition from continua to discontinua. When the damage variable $d$ reaches 1 , the zero-thickness CIEs are completely damaged. The completely damaged CIEs are deleted and the contact surfaces of the neighboring continuum FEs are simultaneously activated. The solid medium can be divided into pieces of deformable bodies by removal of completely damaged CIEs. The deformable bodies, comprising continuum FEs and CIEs with or without minor damage, interact with each other according to the contact surfaces and can further fracture and fragment.

A hard contact model [24] is adapted for the interaction between the deformable bodies. When surfaces of the deformable bodies are in contact, there is no limit to the magnitude of contact pressure that can be transmitted between them. The contact surfaces separate whether the contact pressure reduces to zero. The hard contact model minimizes the overlap of the contact surfaces and does not allow the transfer of tensile stress across the interface. Coulomb friction is considered for shear interaction when surfaces are in contact. Two contacting surfaces can carry shear stresses up to a certain magnitude across their interface before they start sliding relative to one another. The critical shear stress $\tau_{\text {crit }}$ at which sliding of the contacting surfaces starts is defined as

$$
\tau_{\text {crit }}=\mu p,
$$

where $\mu$ denotes the friction coefficient and $p$ is the contact pressure between the contacting surfaces.

\section{Collapse Modeling of Meihua Masonry Arch Dam}

Meihua arch dam, located in Fujian Province of China, was built of cement mortar stones. It was completed in June, 1981. The arch dam was $22 \mathrm{~m}$ high, while $1.2 \mathrm{~m}$ thick at the dam's bottom. A concrete pedestal was set up between the foundation and the dam body. A layer of asphalt was daubed on the interface between the concrete pedestal and the cement-mortar-stone dam body, so-called peripheral joint, resulting in small friction coefficient across the interface.

The reservoir was filled to its normal operation level at the construction completion. The normal operation reservoir level was $49.5 \mathrm{~m}$ and the corresponding water depth was $21.3 \mathrm{~m}$. At the time of the torrential rain on 20 July 1981, the reservoir level rose rapidly resulting in dam overtopping, whereas there were not any signs of endangering of the safety of the dam. Two months later, at 13:35 on 18 September, the arch dam with normal operation reservoir level suddenly fails and collapses, releasing all the reservoir water. The rush of water destroyed some farmlands downstream but, mercifully, did not cause loss of human life.

Figure 4 shows the scene of the collapsed arch dam. The dam body above the concrete pedestal fragmented into pieces 

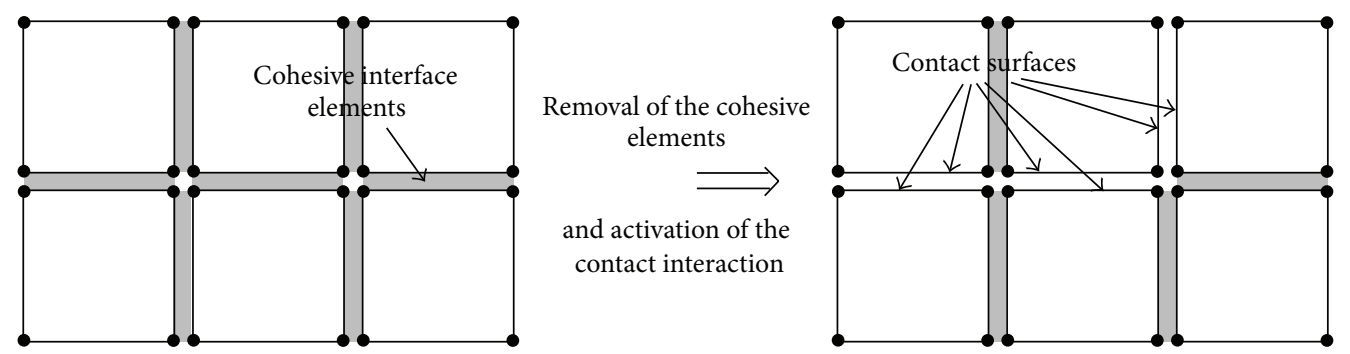

FIgURE 3: Removal of the CIEs and activation of the contact interaction when the CIEs are completely damaged.

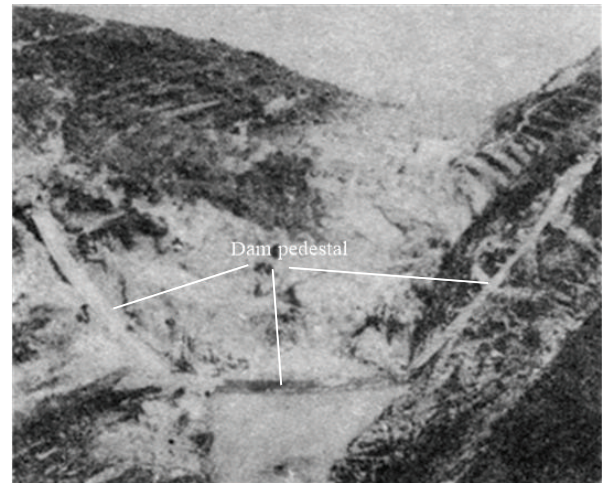

(a) Upstream view

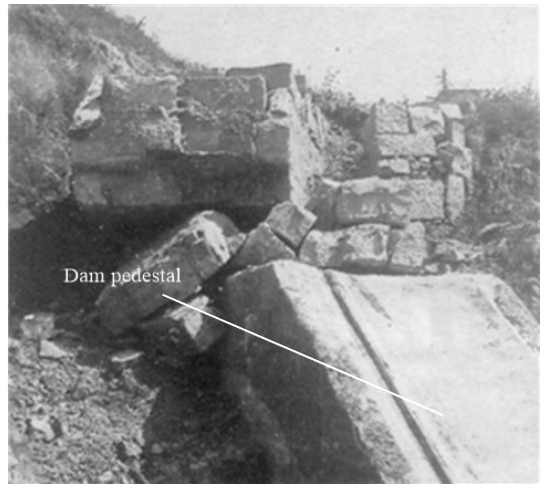

(b) Left abutment

FIGURE 4: Scene of the Meihua masonry arch dam after its collapse.

of cement mortar stones. The cement mortar stones were washed away down the river, whereas the concrete pedestal remained intact after the catastrophe.

Researchers have attempted to find the cause of the collapse of Meihua arch dam, and continuum FE approach and discrete element method were used [25-27]. Herein, the FE approach with CIEs is applied to simulate the continuum behavior and the subsequent collapse process of Meihua arch dam.

3.1. FE Discretization of the Dam and Material Properties. The masonry arch dam and the concrete pedestal are discretized into 8-node brick elements. The element size of the dam is about $1.0 \mathrm{~m}$ in both the vertical and cross-stream directions. Three layers of elements are assigned across the dam thickness. CIEs are inserted between all the interfaces of the brick elements of the dam body. The masonry arch dam is initially in contact with the concrete pedestal. In this study, only static loads, including gravity and hydrostatic pressure, are considered. The model with part of foundation can provide sufficiently accurate results, and thus the whole foundation is neglected to save computational time. The FE mesh of the simulated model is shown in Figure 5.

The following mechanical properties of cement mortar stones are used in this analysis: Young's modulus $E_{m}=$ $8 \times 10^{3} \mathrm{MPa}$, Poisson's ratio $v_{m}=0.3$, and density $\rho_{m}=$ $2360 \mathrm{~kg} / \mathrm{m}^{3}$. The CIEs describe fracture of the dam. The initial stiffness of the CIEs can be selected as $E_{c}=20 E_{m}=160 \times$ $10^{3} \mathrm{MPa}$ so as to obtain a structural response with sufficient

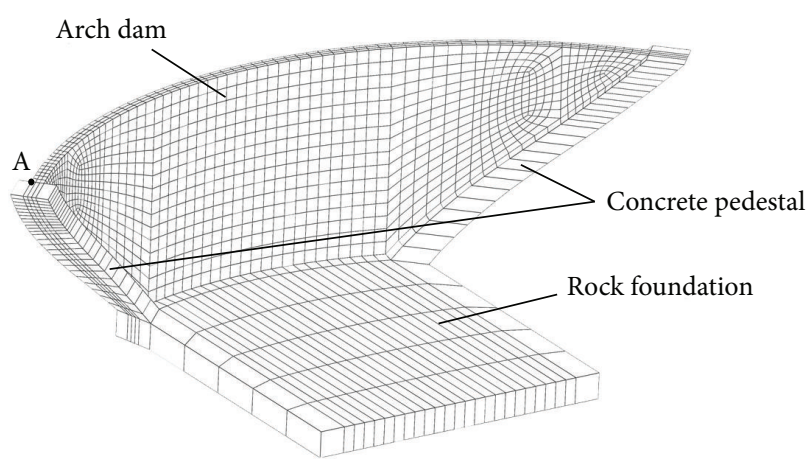

FIGURE 5: FE mesh of the arch dam.

accuracy. The purely tensile strength of the CIEs is $t_{n 0}=$ 1.0 $\mathrm{MPa}$, while the purely shear strength is $t_{s 0}=t_{p 0}=$ 0.6 MPa. When CIEs are completely damaged and deleted, the friction coefficient between the brick elements that may become in contact is taken as $\mu_{c}=1.0$. The mechanical properties of the concrete pedestal and the rock foundation are taken as Young's modulus $E_{f}=24 \times 10^{3} \mathrm{MPa}$, Poisson's ratio $v_{f}=0.2$, and density $\rho_{f}=2450 \mathrm{~kg} / \mathrm{m}^{3}$.

The friction coefficient between the dam body and the concrete pedestal is an important parameter that affects the behavior of the arch dam. Four cases with different friction coefficients, that is, $\mu_{j}=1.0,0.9,0.8$, or 0.7 , are performed.

If full loads are immediately applied at the beginning of an explicit dynamic analysis, the structure would be likely 


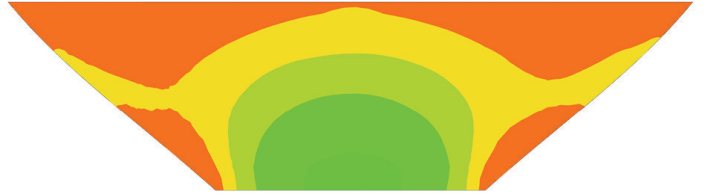

Continuum model

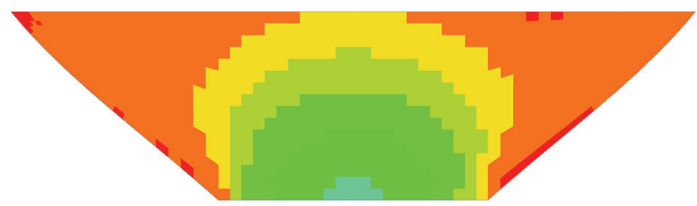

FE approach with CIEs

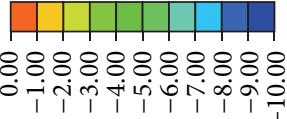

(a) Upstream face

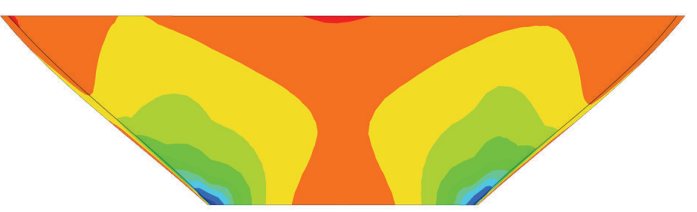

Continuum model

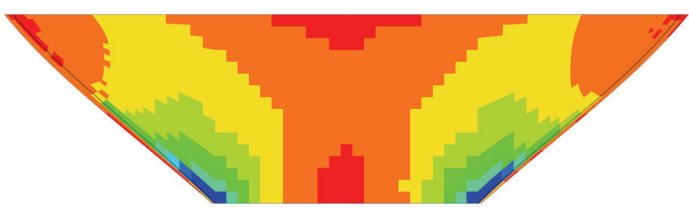

FE approach with CIEs

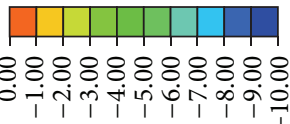

(b) Downstream face

FIGURE 6: Minimum principal stress distribution in the dam with friction coefficient $\mu_{j}=1.0$ across the peripheral joint.
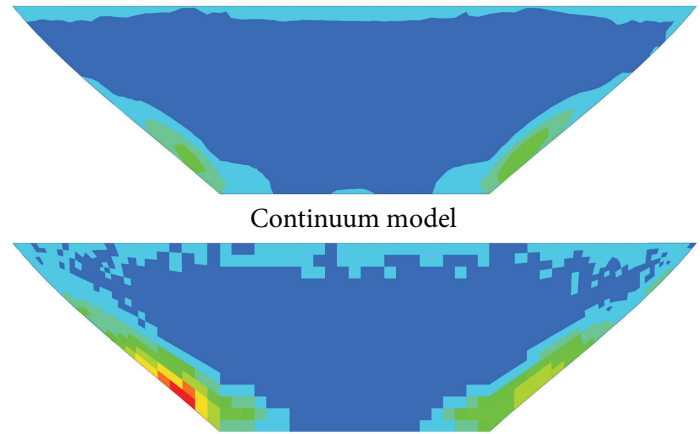

FE approach with CIEs

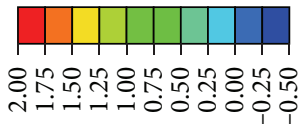

(a) Upstream face
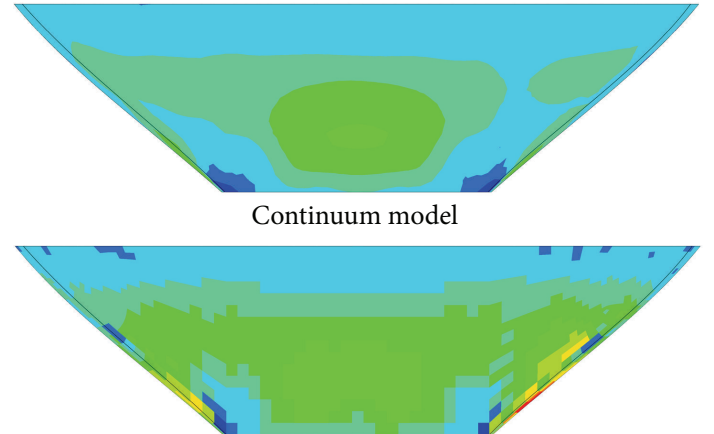

FE approach with CIEs

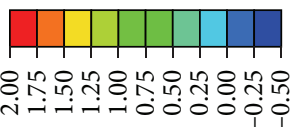

(b) Downstream face

FIGURE 7: Maximum principal stress distribution in the dam with friction coefficient $\mu_{j}=1.0$ across the peripheral joint.

to sustain an impact loading and out of consistency with reality when the dam is under hydrostatic pressure. Therefore, the hydrostatic pressure applied to the arch dam is gradually increased from 0 to the normal reservoir level in $2 \mathrm{~s}$ in order to achieve a quasi-static loading process in the explicit dynamic analysis. A period of $10 \mathrm{~s}$ is calculated in the analysis.

3.2. Numerical Results. The behavior of the masonry arch dam is analyzed using the traditional FE model (continuum model) and the FE approach with CIEs for comparison. The friction coefficient across the peripheral joint is assumed to be 1.0, and the results from both analyses show that the dam remains stable subjected to the hydrostatic pressure. Figure 6 shows the minimum principal stress distribution in the dam body, while Figure 7 illustrates the maximum part. The analyses using the continuum model and the FE approach with CIEs achieve similar stress distributions, as well as close peak stress values. It demonstrates that the FE approach with CIEs provides accurate response of the dam before it fails.

High compression stress zone appears on the upstream face above the dam heel and on the downstream face near the peripheral joint. The peak value of compression stress is about 10.0 MPa that may not reach the compression strength of the cement mortar stones. High tensile stress region occurs on the peripheral joint and on the bottom portion of the downstream face with the peak value exceeding 1.0 MPa, and thus fracture is expected to form there.

Vertical displacement of point $\mathrm{A}$ on the right abutment of the dam is a vital index for monitoring stability of Meihua dam subjected to hydrostatic pressure. The variation of vertical displacements at point $\mathrm{A}$ is shown in Figure 8. 

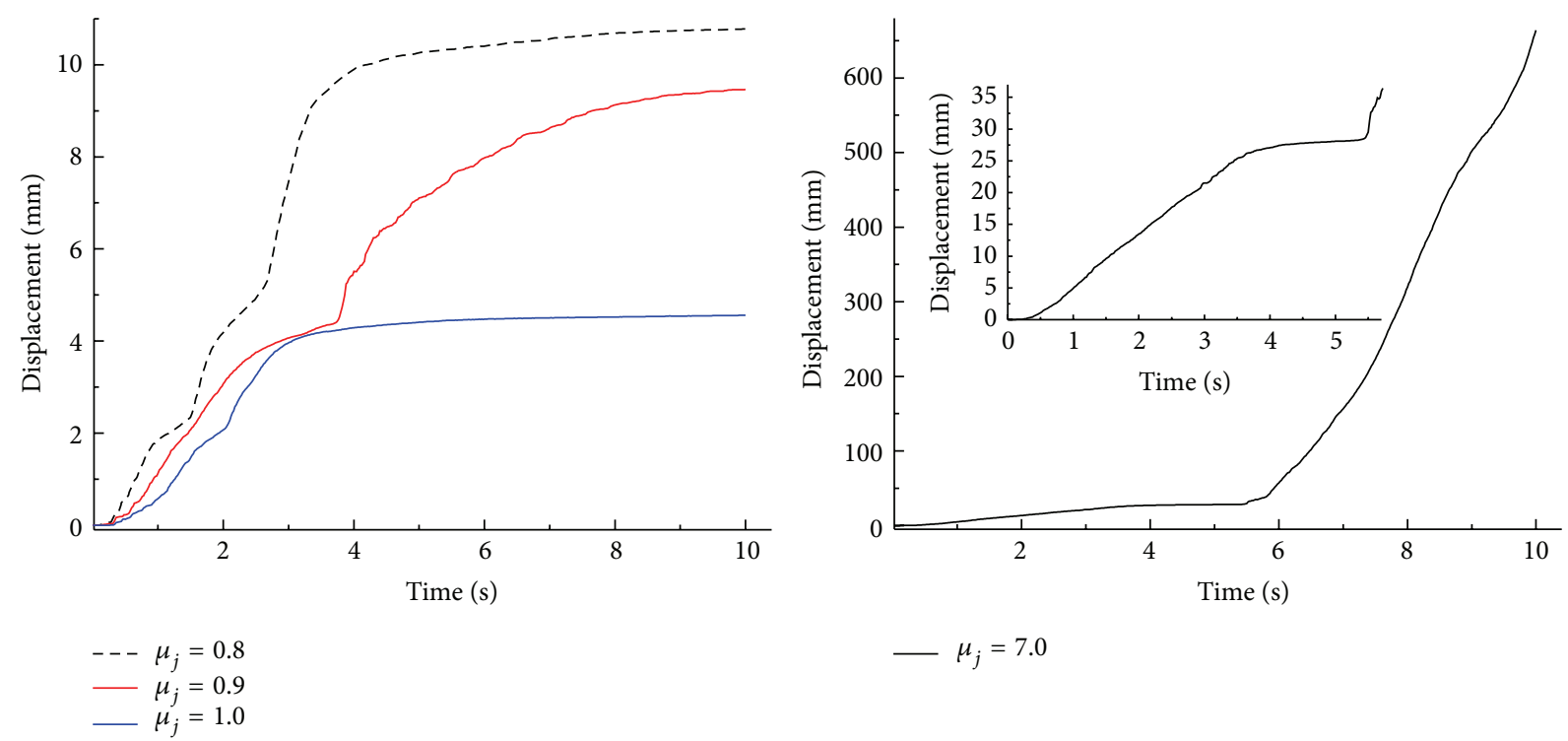

$-\mu_{j}=7.0$

FIGURE 8: Vertical displacements of the dam abutment with different friction coefficients across the peripheral joint.

Influence of friction coefficient across the peripheral joint on the dam response is examined. In the cases when the friction coefficient is larger than 0.7 , that is, $\mu_{j}=0.8,0.9$, or 1.0, the arch dam slides upwards along the peripheral joint but it remains stable with no fracture in the dam body. The vertical displacements for these cases finally approach $10.8 \mathrm{~mm}, 9.5 \mathrm{~mm}$, and $4.6 \mathrm{~mm}$, respectively. In contrast, if the friction coefficient reduces to 0.7 , the arch dam slides upwards slowly during loading process. The vertical displacement reaches $13.5 \mathrm{~mm}$ at the end of loading and then gradually increases to $27.2 \mathrm{~mm}$ in the next $2 \mathrm{~s}$. A rapid increase of the vertical displacement occurs at $t=5.5 \mathrm{~s}$, implying that the dam becomes unstable.

The transferring of the arch dam from continua to discontinua, that is, the collapse process, is illustrated in Figure 9. Vertical cracking first occurs on the downstream face at the base of the dam, which is the high tensile stress region under hydrostatic pressure. These vertical cracks propagate upwards reaching the dam crest and meanwhile penetrate the dam body from the downstream face to the upstream face. The arch action of the dam is weakened, and the external forces previously borne by the arches are transferred to the cantilevers of the dam, resulting in horizontal cracks in the dam near the peripheral joint. The bottom portion of the dam is separated into blocks by intersection of the cracks. These separated blocks move towards downstream under the hydrostatic pressure. Simultaneously, the upper portion of the dam body above the separated blocks is falling with an opening of a middle vertical crack. The dam body finally fragments into pieces of blocks, which would be washed away by the running reservoir water.

The failure mechanism of the Meihua masonry arch dam can be obtained based on the analysis. The layer of asphalt daubed on the peripheral joint reduces the shear strength between the concrete pedestal and the dam body. With low sliding resistance strength, the dam body may slide upward along the peripheral joint due to hydrostatic pressure, leading to weakening of the arch action and fracturing of the dam. The failure mechanism is reasonable compared with the field observation after the dam collapse.

Peripheral joint may change the distribution patterns of stress in arch dams. The peripheral joint improves the safety of the dam-foundation system in some cases such as Dez Dam [28], while it is the cause of collapse of Meihua dam. Therefore, if an arch dam is set up with a peripheral joint, it is necessary to access the influence on safety of the damfoundation system.

\section{Conclusions}

A finite element (FE) approach with cohesive interface elements (CIEs) is presented to simulate collapse of continuum structures. The element removal technique merged with the general contact algorithm is adapted in the FE approach to achieve modeling for a transition from continua to discontinua, that is, fracture, fragmentation, and collapse. Collapse process and failure mechanism of Meihua masonry arch dam are investigated using the presented procedure. Regarding the collapse process of the dam body, vertical cracking first occurs on the downstream face at the base of the dam and propagates upwards reaching the dam crest under the hydrostatic pressure of normal operation reservoir level. Subsequently, horizontal cracks form in the dam near the peripheral joint. The bottom portion of the dam is separated into blocks by intersection of these cracks, and they move towards downstream. At the same time, the upper portion of the dam body above the separated blocks falls with an opening of a middle vertical crack. The masonry dam body finally fragments into pieces of blocks which would be washed away. The collapse process is in good agreement with the field observation after the dam failure. The failure of Meihua arch dam can be attributed to reducing shear strength of the peripheral joint by daubing a layer of asphalt 


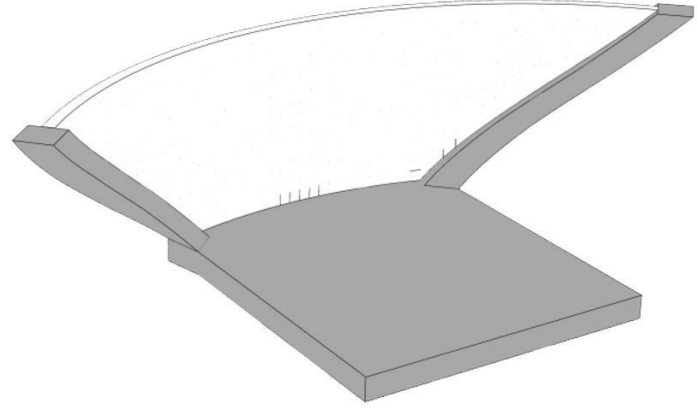

(a) $t=5.3 \mathrm{~s}$

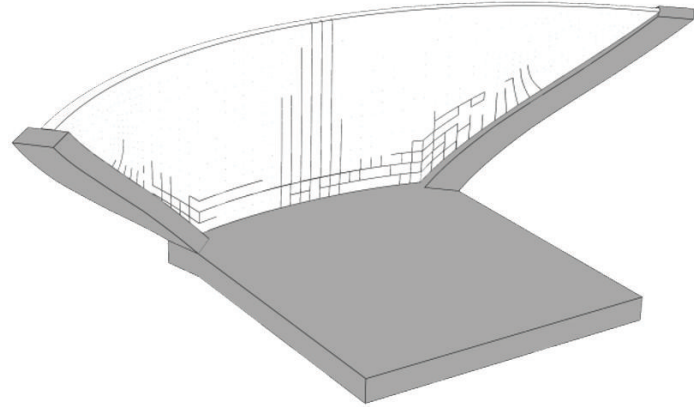

(c) $t=6.0 \mathrm{~s}$

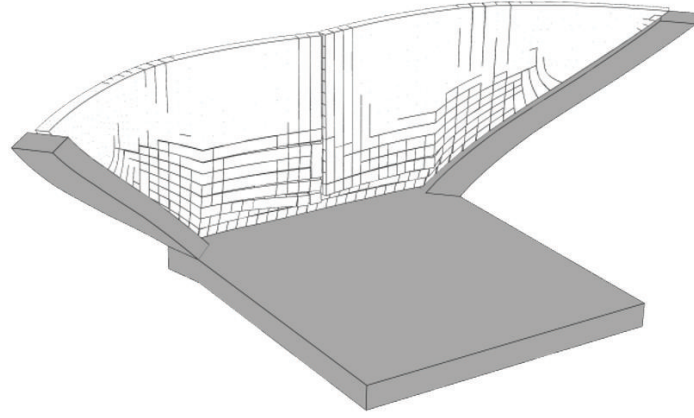

(e) $t=8.6 \mathrm{~s}$

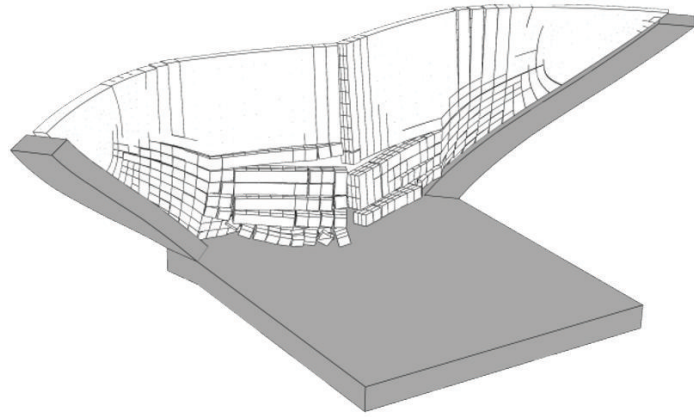

(g) $t=9.3 \mathrm{~s}$

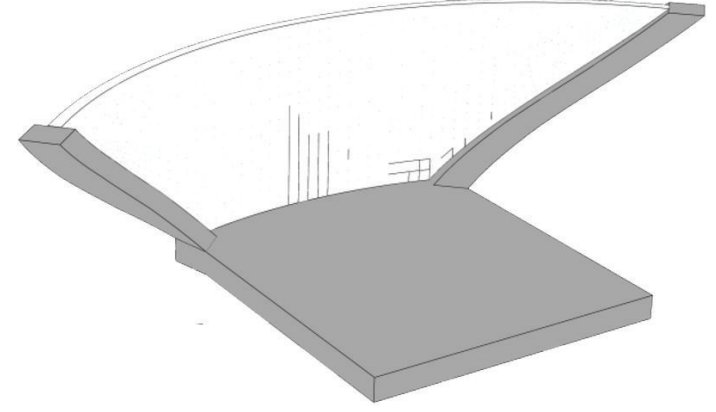

(b) $t=5.5 \mathrm{~s}$

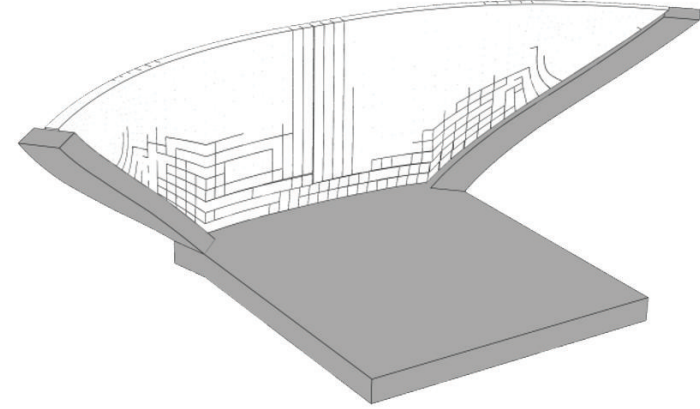

(d) $t=7.4 \mathrm{~s}$

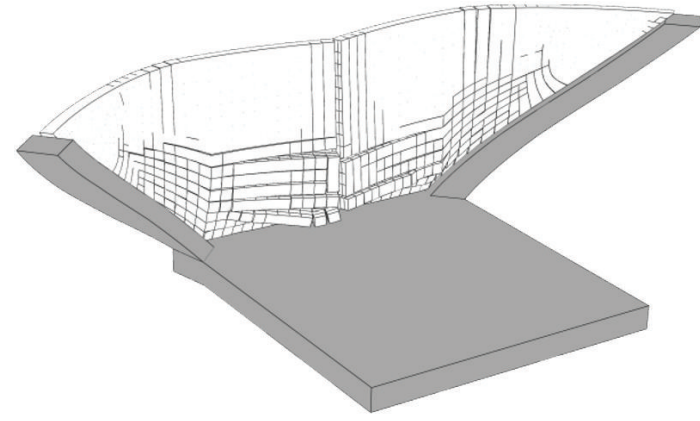

(f) $t=9.0 \mathrm{~s}$

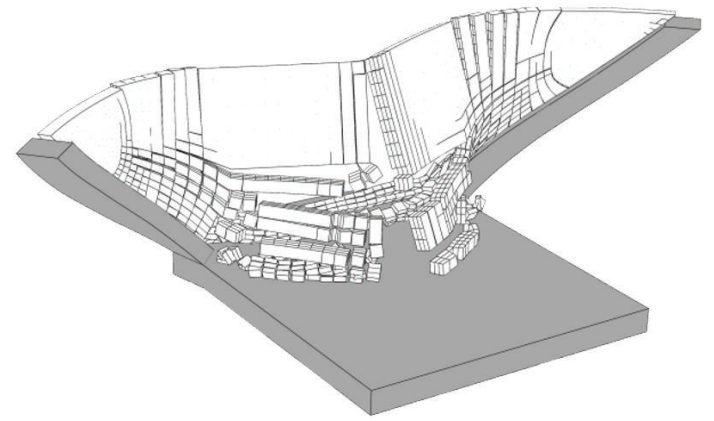

(h) $t=10.0 \mathrm{~s}$

FIGURE 9: Collapse process of the dam with friction coefficient $\mu_{j}=0.7$ across the peripheral joint.

there. With low sliding resistance strength, the masonry dam body may slide upwards along the peripheral joint due to hydrostatic pressure, leading to weakening of the arch action, fracturing, and final collapse of the dam.

\section{Conflict of Interests}

The author declares that there is no conflict of interests regarding the publication of this paper. 


\section{Acknowledgments}

The author gratefully acknowledges the support for this investigation from the National Natural Science Foundation of China (Grant no. 51209120) and the Tsinghua University Initiative Scientific Research Program (Grant no. 20131089285).

\section{References}

[1] S. Valliappan, M. Yazdchi, and N. Khalili, "Seismic analysis of arch dams-a continuum damage mechanics approach," International Journal for Numerical Methods in Engineering, vol. 45, no. 11, pp. 1695-1724, 1999.

[2] Y. Calayir and M. Karaton, "A continuum damage concrete model for earthquake analysis of concrete gravity damreservoir systems," Soil Dynamics and Earthquake Engineering, vol. 25, no. 11, pp. 857-869, 2005.

[3] H. Zhong, G. Lin, X. Li, and J. Li, "Seismic failure modeling of concrete dams considering heterogeneity of concrete," Soil Dynamics and Earthquake Engineering, vol. 31, no. 12, pp. 16781689, 2011.

[4] J. Pan, Y. Xu, and F. Jin, "Seismic performance assessment of arch dams using incremental nonlinear dynamic analysis," European Journal of Environmental and Civil Engineering, vol. 19, no. 3, pp. 305-326, 2014.

[5] J. Pan, C. Zhang, J. Wang, and Y. Xu, "Seismic damagecracking analysis of arch dams using different earthquake input mechanisms," Science in China, Series E: Technological Sciences, vol. 52, no. 2, pp. 518-529, 2009.

[6] J. Pan, Y. Feng, Y. Xu, F. Jin, C. Zhang, and B. Zhang, "Chemodamage modeling and cracking analysis of AAR-affected concrete dams," Science China Technological Sciences, vol. 56, no. 6, pp. 1449-1457, 2013.

[7] J. Pan, Y. T. Feng, F. Jin, and C. Zhang, "Numerical prediction of swelling in concrete arch dams affected by alkali-aggregate reaction," European Journal of Environmental and Civil Engineering, vol. 17, no. 4, pp. 231-247, 2013.

[8] M. Shi, H. Zhong, E. T. Ooi, C. Zhang, and C. Song, "Modelling of crack propagation of gravity dams by scaled boundary polygons and cohesive crack model," International Journal of Fracture, vol. 183, no. 1, pp. 29-48, 2013.

[9] S.-N. Roth, P. Léger, and A. Soulaïmani, "A combined XFEMdamage mechanics approach for concrete crack propagation," Computer Methods in Applied Mechanics and Engineering, vol. 283, pp. 923-955, 2015.

[10] J. Pan, Y. Feng, F. Jin, C. Zhang, and D. R. J. Owen, "Comparison of different fracture modelling approaches to gravity dam failure," Engineering Computations, vol. 31, no. 1, pp. 18-32, 2014.

[11] O. A. Pekau and C. Yuzhu, "Failure analysis of fractured dams during earthquakes by DEM," Engineering Structures, vol. 26, no. 10, pp. 1483-1502, 2004.

[12] D. S. Dugdale, "Yielding of steel sheets containing slits," Journal of the Mechanics and Physics of Solids, vol. 8, no. 2, pp. 100-104, 1960.

[13] G. I. Barenblatt, "The mathematical theory of equilibrium cracks in brittle fracture," in Advances in Applied Mechanics, Vol. 7, pp. 55-129, Academic Press, New York, NY, USA, 1962.

[14] A. Hillerborg, M. Modéer, and P.-E. Petersson, "Analysis of crack formation and crack growth in concrete by means of fracture mechanics and finite elements," Cement and Concrete Research, vol. 6, no. 6, pp. 773-781, 1976.
[15] H. R. Lotfi and P. B. Shing, "Interface model applied to fracture of masonry structures," Journal of Structural Engineering, vol. 120, no. 1, pp. 63-80, 1994.

[16] J. Cervenka, J. M. Chandra Kishen, and V. E. Saouma, "Mixed mode fracture of cementitious bimaterial interfaces; part II: numerical simulation," Engineering Fracture Mechanics, vol. 60, no. 1, pp. 95-107, 1998.

[17] V. Saouma, "Applications of fracture mechanics to cementitious materials; a personal perspective," in ACI SP-300 Fracture Mechanics Applications in Concrete, G. Cusatis, Ed., American Concrete Institute, 2015.

[18] G. T. Camacho and M. Ortiz, "Computational modelling of impact damage in brittle materials," International Journal of Solids and Structures, vol. 33, no. 20-22, pp. 2899-2938, 1996.

[19] A. Pandolfi, P. Krysl, and M. Ortiz, "Finite element simulation of ring expansion and fragmentation: the capturing of length and time scales through cohesive models of fracture," International Journal of Fracture, vol. 95, no. 1-4, pp. 279-297, 1999.

[20] X. P. Xu and A. Needleman, "Numerical simulations of fast crack growth in brittle solids," Journal of the Mechanics and Physics of Solids, vol. 42, no. 9, pp. 1397-1434, 1994.

[21] J. H. Song, H. Wang, and T. Belytschko, "A comparative study on finite element methods for dynamic fracture," Computational Mechanics, vol. 42, no. 2, pp. 239-250, 2008.

[22] V. P. Nguyen, "An open source program to generate zerothickness cohesive interface elements," Advances in Engineering Software, vol. 74, pp. 27-39, 2014.

[23] P. P. Camanho and C. G. Davila, "Mixed-mode decohesion finite elements for the simulation of delamination in composite materials," Tech. Rep. NASA/TM-2002-211737, 2002.

[24] S. Dassault, Abaqus 6.12 Theory Manual, 2012.

[25] C. Du and Y. Qiu, "Analysis on the failure types of Meihua arch dam with peripheral joints," Water Power, vol. 36, no. 5, pp. 6163, 2002.

[26] Y. Cui, C. Zhang, Y. Xu, F. Jin, and G. Wang, "Failure simulation of the Meihua arch dam," Journal of Tsinghua University (Science \& Technology), vol. 42, pp. 88-92, 2002.

[27] C. Zhang and F. Jin, "Three-dimensional modal deformable discrete element method and its application study," Design of Hydroelectric Power Station, vol. 26, no. 3, pp. 1-9, 2010.

[28] M. A. Hariri-Ardebili, H. Mirzabozorg, and M. Ghaemian, "Pulvino and peripheral joint effects on static and seismic safety of concrete arch dams," Scientia Iranica, Transactions A: Civil Engineering, vol. 20, no. 6, pp. 1579-1594, 2013. 


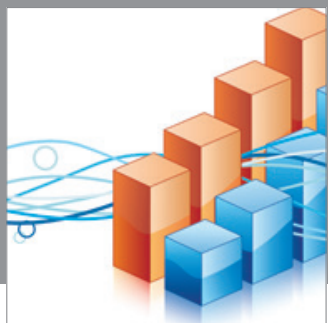

Advances in

Operations Research

mansans

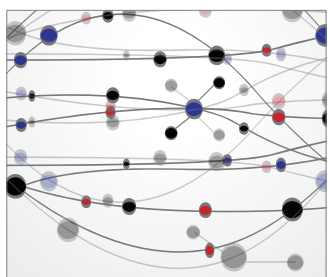

The Scientific World Journal
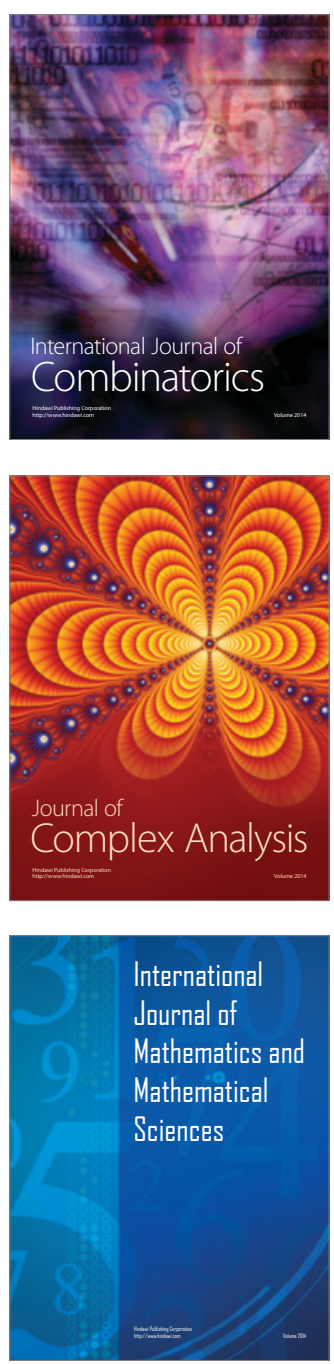
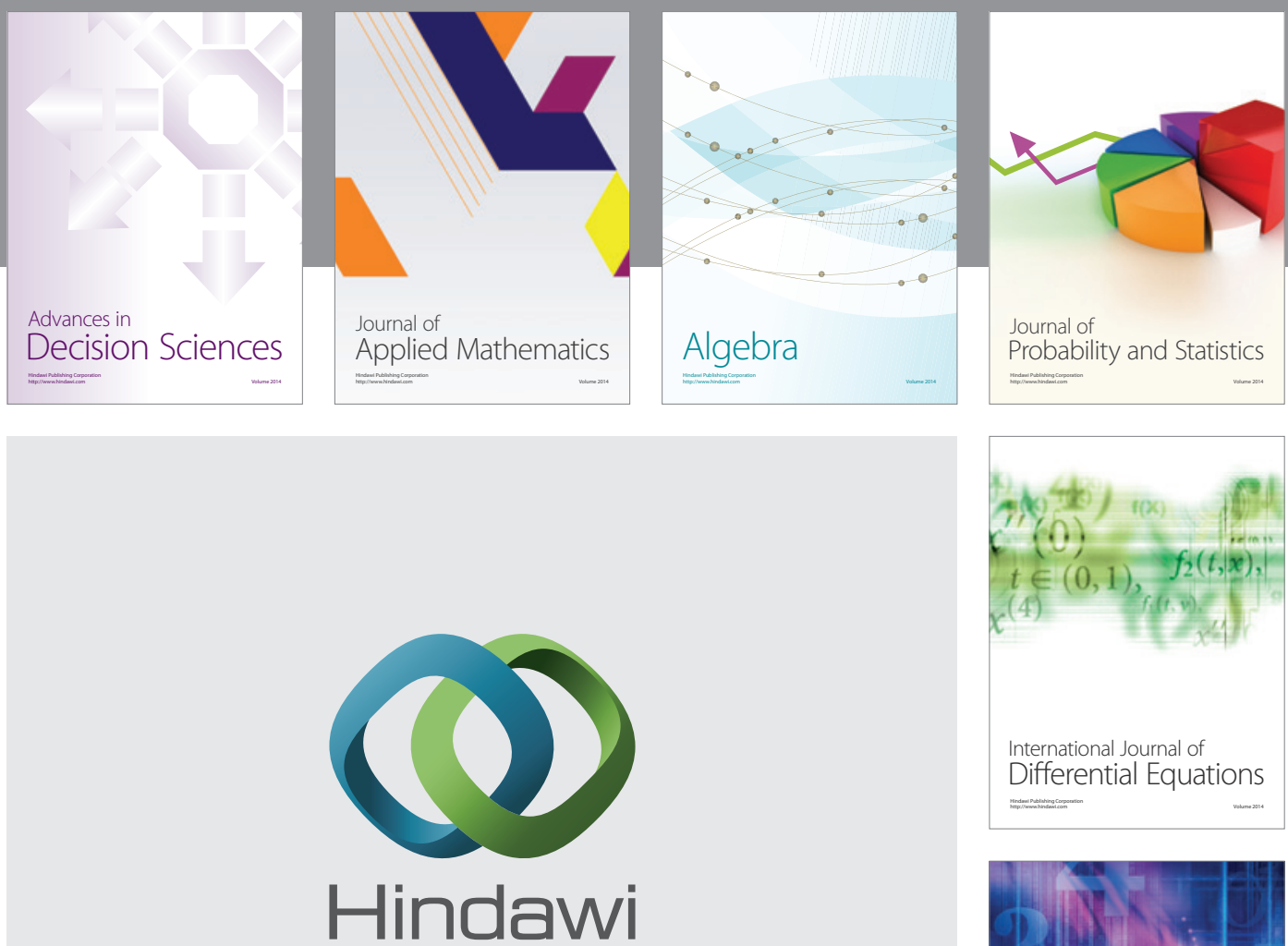

Submit your manuscripts at http://www.hindawi.com
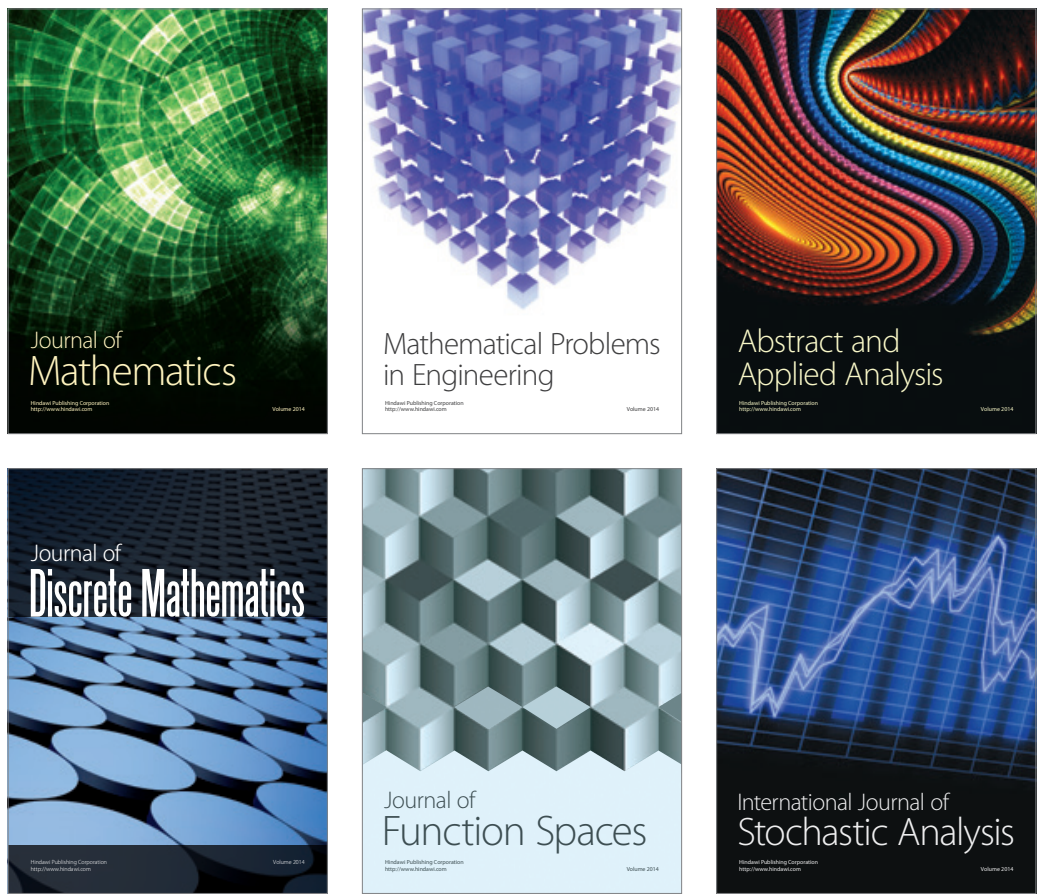

Journal of

Function Spaces

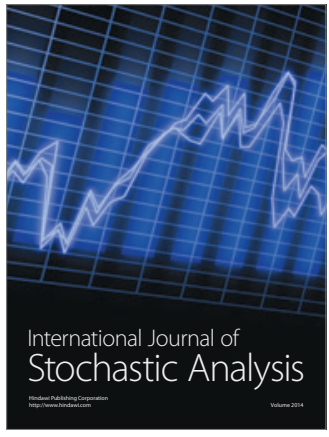

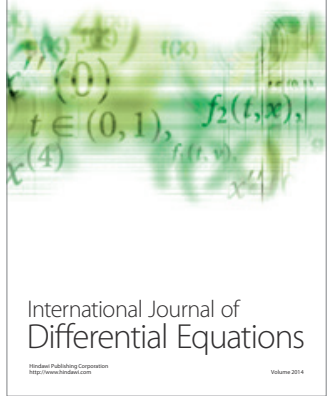
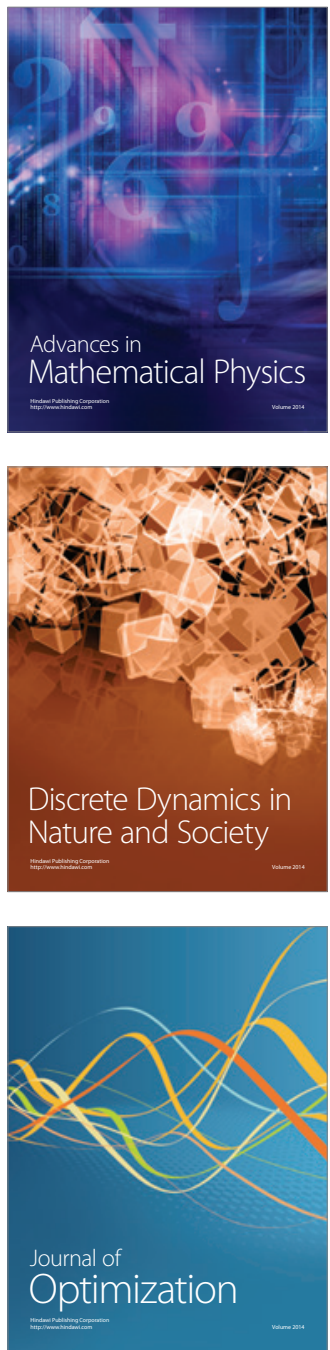